\title{
EchoFakeD: improving fake news detection in social media with an efficient deep neural network
}

\author{
Rohit Kumar Kaliyar ${ }^{1} \cdot$ Anurag Goswami $^{1} \cdot$ Pratik Narang $^{2}$ \\ Received: 20 May 2020 / Accepted: 11 December 2020 / Published online: 2 January 2021 \\ (C) The Author(s), under exclusive licence to Springer-Verlag London Ltd. part of Springer Nature 2021
}

\begin{abstract}
The increasing popularity of social media platforms has simplified the sharing of news articles that have led to the explosion in fake news. With the emergence of fake news at a very rapid rate, a serious concern has produced in our society because of enormous fake content dissemination. The quality of the news content is questionable and there exists a necessity for an automated tool for the detection. Existing studies primarily focus on utilizing information extracted from the news content. We suggest that user-based engagements and the context related group of people (echo-chamber) sharing the same opinions can play a vital role in the fake news detection. Hence, in this paper, we have focused on both the content of the news article and the existence of echo chambers in the social network for fake news detection. Standard factorization methods for fake news detection have limited effectiveness due to their unsupervised nature and primarily employed with traditional machine learning models. To design an effective deep learning model with tensor factorization approach is the priority. In our approach, the news content is fused with the tensor following a coupled matrix-tensor factorization method to get a latent representation of both news content as well as social context. We have designed our model with a different number of filters across each dense layer along with dropout. To classify on news content and social context-based information individually as well as in combination, a deep neural network (our proposed model) was employed with optimal hyper-parameters. The performance of our proposed approach has been validated on a real-world fake news dataset: BuzzFeed and PolitiFact. Classification results have demonstrated that our proposed model (EchoFakeD) outperforms existing and appropriate baselines for fake news detection and achieved a validation accuracy of 92.30\%. These results have shown significant improvements over the existing state-of-the-art models in the area of fake news detection and affirm the potential use of the technique for classifying fake news.
\end{abstract}

Keywords Echo chamber $\cdot$ Deep learning $\cdot$ Social media $\cdot$ Fake news $\cdot$ Tensor decomposition

\section{Introduction}

In today's digital era, sharing articles and interacting with people over social media is just a click away. Due to rapid growth in internet technology, users can share information online via a Smartphone where social media has become a

Pratik Narang

pratik.narang@pilani.bits-pilani.ac.in

Rohit Kumar Kaliyar

rk5370@bennett.edu.in

Anurag Goswami

anurag.goswami@bennett.edu.in

1 Department of Computer Science Engineering, Bennett University, Greater Noida, India

2 Department of CSIS, BITS Pilani, Pilani, Rajasthan, India popular and quick source for sharing news articles [1]. It has created many challenges for researchers to find the exact origin of the news articles based on geographical location [1]. This flexibility and ease of circulating information on social media have led to an increase in the number of fake stories published to mislead society's ordinary people (individuals and organization-based). These false stories are called fake news. The goal of creating fake news is to appear as credible information that 


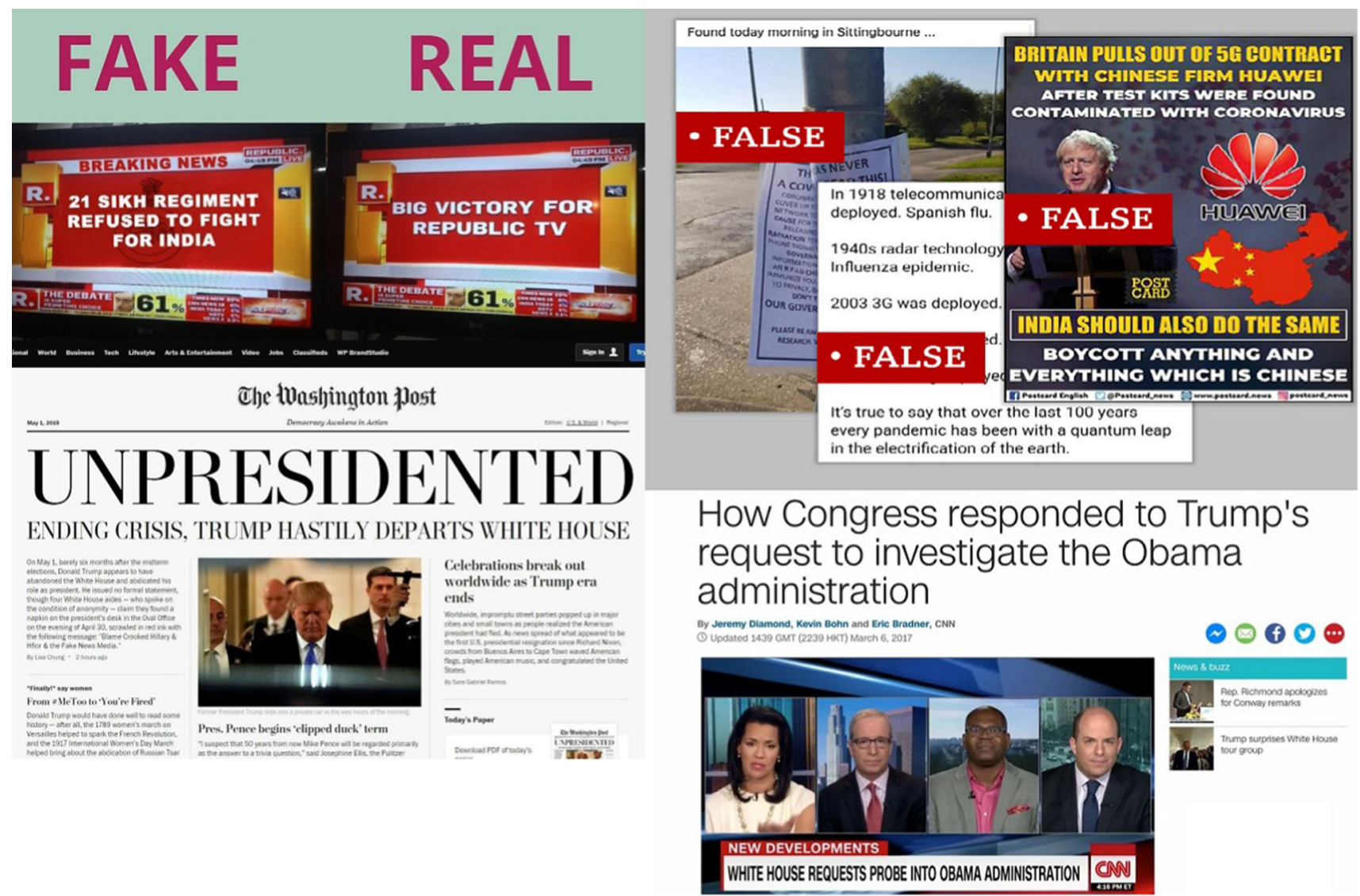

Fig. 1 Examples of fake news on social media. Source: Facebook and Twitter

ends in a quick share on social media. It is evident that due to sharing extensive volume information $[1,2]$ daily with quality of content loses the truth's ground. The objective of spreading such information is to manipulate the public opinion for financial and political gains [3]. Fake news has also shown adverse effects on stock-prices, and large infrastructure investments [4]. One such example is about a bomb explosion-based news $[5,6]$ in which former US President Barack Obama had injured. This news annihilated 130 billion in the stock market within a few minutes.

The complete life cycle of fake news starting from creation until propagation has been described by Kumar et al. [7]. In this life cycle, the role of individuals (user, creator, publisher, etc.) has been investigated with the overall structure [7] of a fake news article. We can categories the stages of fake news into three forms: creation (how a piece of fake news is created), the publication (publication and feedback of a fake article), and propagation (how a piece of fake news is shared among users in social media). Few examples of fake news have shown with the help of Fig. 1 during COVID-19 and the 2016 US General Election. The main contributors to fake news are Fakesters. "Fakesters" spread fake news with specific plans to deceive people and publish fabricated articles online by distorting the fact behind it. Main stakeholders for fighting against fake news are fact-checking organizations like Snopes ${ }^{1}$ and Politifact ${ }^{2}$ etc. These organizations are of value to validate the news content with facts-based methods. However, for checking the quality of content, these methods are not automated and tend to be very timeconsuming. It is also difficult to examine the quality of content daily via these techniques for news created by different Fakesters.

To check the quality of content for fake news detection, we need to extract useful features (refer Fig. 2 for more details) from social media datasets $[1,7,8]$. There exist a few datasets for fake news detection; most of them contain only linguistic features. Few of them contain semantic and social contexts-based features. News fabrication is mostly happening with textual content. Existing learning techniques for fake news detection can be generally categorized as (1) news content-based learning, where the main focus is on the news articles, way of writing the malicious news, linguistic styles, etc., and (2) social context-based learning,

\footnotetext{
1 https://www.snopes.com.

2 https://www.politifact.com.
} 
Fig. 2 Features for fake news detection

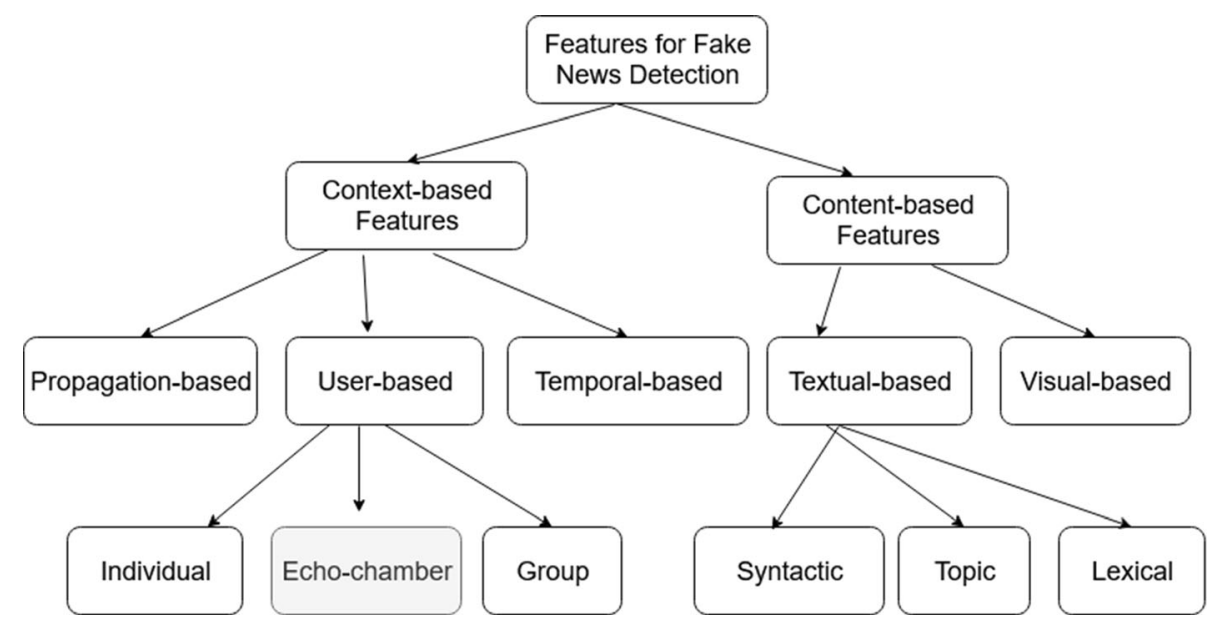

where the main focus on user-related behaviours (a type of users, comments, likes, share etc.)

News content-based methodologies $[1,9,10]$ primarily focus on extracting different features from fake news articles, including both content-based as well as stylebased. Information-based methodologies [9, 11] plan to utilize external sources to certainty check the honesty of the claims in news content. Furthermore, fake news publishers regularly have malignant plans to spread deluding news, requiring specific composition styles to interest and convince a wide variety of consumers that are not found in real news stories. Style-based methodologies $[9,11]$ mainly focus on the writing style of manipulators and creators for the context of fake news. It is evident that for efficient fake news detection, content-based methodologies are alone not sufficient. We need to investigate the fake news articles with social context-based methods.

Social context-based methodologies [9, 12, 13] deals with the relationship among users, news article, and related publishers. These methodologies are efficient in recognizing fake news articles. Social context provides valuable information about users-based interaction with both the fake news and the real news. Features related to fake news detection are shown in Fig. 2. Instance-based methodologies $[13,14]$ are helpful to know the perspectives of users from a sharing news article to induce the integrity of unique news stories. Furthermore, propagation-based methodologies [13] (how a piece of news is shared among users using diverse context-related social media platforms) are based on the relations of significant social media posts to guide the learning of validity scores by propagating credibility values [13] between users, posts, and news.

In the era of computing, at any social media platform, a user is always connected to a specific group of peoples having the same mindset or liking is called a user-community. These user communities can be an essential factor for fake news classification due to their common perception about sharing articles. They are a group of users with the same interests in social circles where opposing ideas are rejected and disapproved by the majority. For example, when looking at comments to a user post on Facebook, many of the comments that agree with the post reflect one type of echo chamber. This agreement, combined with the others (e.g. number of likes, shares), may lead the user to get a false impression that the information presented is correct. Moreover, Facebook, by default, increases the ranking [14] of the comments based on the number of replies and likes received by the user's friends. Based on the issues discussed above, we were motivated to design an efficient deep learning model utilizing social context-based features.

\subsection{Motivation and research goal}

Fake news detection has earned huge attention from researchers across the world. Social media platforms have become a popular bridge among users $[15,16]$ for quick and seamless access to fake news. Existing detection methods [17-24] primarily focus on content or social context-based information extracted from news articles. Despite receiving huge attention from the leading research communities, the necessity of an efficient detection model still exists with the capability to handle not only content but also context and community-level features with a tensor-factorization method.

Research goal To build an effective deep learning model for the detection of fake news utilizing the social context of news articles.

In this research, we propose an effective deep learning model with a combination of both content and contextrelated features. In one of the existing research, Gupta et al. [17] have explored the problem of fake news with the user and community-level information using the tensor factorization method. In their approach, two methods (CITDetect 
and CIMTDetect) have been proposed using both content and user-community information as a combination. They experimented with traditional machine learning classifier (SVM) and achieved an accuracy of $81.30 \%$ and $81.80 \%$, respectively, with both real-world fake new datasets: BuzzFeed and PolitiFact. In their approach, information about the dimensionality of features is vague. Deep learning techniques learn by creating a more abstract representation of data as the network grows deeper; thus, the model automatically extracts features and yields higher accuracy results. Keeping these points in mind, our proposed model is one step ahead of the existing approach. In our approach, for textual modality and effective detection, we have performed extensive feature set-based studies to classify fake news (refer to Table 3 for more details). In this paper, the user's engagement with the news articles is captured and fused with user-community interaction to form a 3-mode tensor (content, social-context, and usercommunity information). This tensor is capable of handling multi-relational data [25] and provides a higher dimensional generalization of matrices. Tensor factorization decomposes the higher-order tensor into low-rank tensors. The resulting low-rank tensors capture the complex relations between the objects representing the help of models of the tensor. We achieved the dimension of a combined matrix (content-context information) is $182 \times 1503$, in which many news stories are 182 , and the size of the input word embedding is 1503. Standard factorization methods [26] have limited effectiveness due to their unsupervised nature. Therefore, in this research, a coupled matrix-tensor factorization method is used with a latent representation of both (news content and context) of news articles. In the coupled matrix-tensor factorization method (also known as CP-decomposition), we have utilized the standard factorization method to decompose the matrix. In the case of labelled data, the class information could help the factorization process to identify fake news better. Then, our proposed network is used for modelling this combined representation of fake news information. A thin deep network [26-28] with two or three hidden layers also outperforms all traditional methods by a significant margin (2-4\%) on handcrafted features. The architecture worked well on small and large datasets and decrease classification error. Deep neural networks eliminate feature engineering and are capable of handling high dimensional datasets with millions of parameters that pass through nonlinear function. Therefore, we have considered five dense layers to make our model deep in nature. After increasing more hidden layers with our neural network, it is likely to overfit the model and, in turn, depreciate accuracy on the test data. Using user community-based features with news content as a large dimensionality tensor, we get the optimal results with a neural network having five hidden layers. To validate the classification performance, the proposed deep learning model (EchoFakeD) is employed with both contents and context-based information. Our model outperforms existing and appropriate baselines for fake news detection and achieved an accuracy of $92.30 \%$. The main contributions of this paper are:

- Performed extensive feature set-based studies for the classification of fake news

- Designed an efficient deep neural network combining the content level features of news articles with user's social engagement (echo-chamber infused) to achieve significant results

- Implemented a tensor factorization-based approach with content as well as context-based information.

- Utilized an echo-chamber infused 3-mode Tensor for higher-dimensional generalization

In this paper, Sect. 1 introduces the current trend of fake news, motivation, and our research contribution. Section 2 discusses the related work. Section 3 discusses our proposed architecture in detail. Sections 4 , and 5 discuss the dataset, experimental details, and results presented, followed by a conclusion in Sect. 6.

\section{Related work}

This section briefly summarizes the existing work in the field of fake news detection [29].

\subsection{Fake news detection using news content- based features}

Ott el al. [18] have investigated a novel method for the detecting of deceptive spam reviews. They have explored POS-tags and word-count as features in their research. They have performed their experiments using an opinion spam dataset and achieved an accuracy of $90 \%$ with their proposed classifier.

Feng et al. [19] have investigated the syntactic stylometry for deception detection. They have also detected deceptive reviews based on Context-Free Grammar (CFG) rules. They have performed their experiments based on hotel review data and achieved an accuracy of $91.20 \%$.

Chen et al. [20] have explored lexical and syntactic features to identify the inherited fabricated content with news articles. They have investigated potential methods for the automatic detection of click-bait. They have suggested in their research that a hybrid approach may produce the best results for fake content detection.

Pérez-Rosas et al. [21] have discussed linguistic differences in fake and legitimate news content. They have also discussed a comparative analysis of the automatic and 
manual identification of fake news. They have performed their experiments based on their linguistic-based approach, achieved an accuracy of $74.00 \%$ with a celebrity-news dataset.

\subsection{Fake news detection using social context- based features}

In this section, social context-based approaches [9, 17] have been discussed. These approaches were capable to handle the features related with the user and it's connected network in social media.

Tacchini et al. [30] have investigated an approach to identify fake news based on its consumer feedback. For the classification, experiments have been conducted using a bipartite network with the user's feedback information (number of likes and dislikes for a news article).

Gupta et al. [22] have investigated the credibility of twitter-based events with their novel approach. They have discussed a detailed comparison of their proposed approach with the existing methods. They have also discussed credibility analysis of news articles and graph-based optimization method. In their research, they have also explored a classification approach extracting user-based, tweetbased, and event-based features. They have also investigated the credibility of users connected in a social network with graph-based optimization for efficient results.

Shu et al. [23] have explored the explainable detection of fake news. They have developed a sentence-comment co-attention sub-network to exploit both news contents and user comments. With their proposed method, achieved with an accuracy of $90.40 \%$ using social-context based features only with PolitiFact dataset.

Shu et al. [14] have investigated a novel approach utilizing social context-based features with content-level information. They have explored a relationship between the user, news articles, and publisher as an embedding approach to find effective results.

Gupta et al. [17] have explored a detection method combining content and context-based features in the form of a tensor. With this tensor, a tensor factorization method has been deployed for the classification of the news article. They have introduced two concepts in their research, news cohort analysis and collaborative news recommendation for fake news detection. They have achieved with an F1score of $81.30 \%$.

\subsection{Deep neural networks for fake news detection}

In this section, deep learning approaches [13, 31] have been discussed. These approaches were capable to extract automatic features and improve classification results.
Ma et al. [32] have explored a neural network utilizing contextual features from news articles. They have also investigated the traditional machine learning algorithms with the performance feedback that require hand-crafted features. They have more focused on deep learning-based methods to achieve better classification results.

Ruchansky et al. [12] have investigated a hybrid model combining the source-characteristics and response of user for a news article. They have divided their method into three modules. In the first one, they have discussed the temporal pattern of both users as well as it's engagement with the news article. They have used RNN as a classifier utilizing lower dimension representation of news articles. In the second module, they have investigated the credit score to a user on social media by deploying a fully connected layer to the network. In the third one, they have combined the vector of the first module with the credit score of the previous module for effective fake news detection.

Yang et al. [33] have investigated their novel convolutional neural network (TI-CNN) with a combination of both text and image-based features using fusion techniques. In their approach, they have done more investigation using visual content of the news article for effective classification with some based content-based features. They have explained explicit features include linguistic features, psychological perspective, lexical diversity, and sentiment score.

Zhang et al. [31] have explored a novel architecture (fake detector) combining the linguistic and writing-based features obtained from a news article for the classification. For experimental purpose, gated recurrent unit (GRU) is used for the extraction of latent features. Further, they have used gated diffusive unit (GDU) combining latent features of news-creators, news articles, and subjects.

Shu et al. [34] have explored the problem of fake news with their proposed model (TriFN). In their approach, it models publisher-news relations and user-news interactions simultaneously for fake news classification. Experiments have been conducted with two real-world fake news datasets (BuzzFeed and PolitiFact) and achieved with an accuracy of $86.40 \%$ and $87.80 \%$, respectively.

Zhou et al. [35] have explored a network-based patterndriven fake news detection approach. In their approach, they have studied the patterns of fake news in social networks, which refer to the news being spread. Experiments on two real-world datasets (BuzzFeed and PolitiFact) and achieved with an accuracy of $83.50 \%$.

Wang et al. [36] have investigated an end-to-end framework named Event Adversarial Neural Network (EANN), which can derive event-invariant features and thus benefit the detection of fake news on newly arrived events. Experiments have been conducted using two real- 
world fake news datasets (Weibo and Twitter) and achieved with an accuracy of $71.50 \%$ and $82.70 \%$, respectively.

Zhou et al. [24] have examined a Similarity-Aware FakE news detection method (SAFE) which investigates multi-modal (textual and visual) information of news articles. Experiments on two real-world datasets (PolitiFact and Gossip-cop) and achieved with an accuracy of $87.40 \%$ and $83.80 \%$, respectively. In our previous work [37], authors have explored an efficient deep convolutional neural network for the detection of fake news utilizing content-based features. Further, a deep neural network with four dense layers is designed [38] utilizing both contents as well as context-based features and achieved state-of-the-art results using real-world fake news dataset: BuzzFeed and PolitiFact. In this current research, our proposed deep neural network is one step ahead as compared to existing methods in terms of performance.

In this article, we have considered the presence of echochambers (communities sharing the same faith or confidence) in the given social network. Further, we have represented news articles as 3-mode tensor (news, user, echochamber). With this information, we propose an enhancement of the above-mentioned methods for fake news detection, by combining the textual information of the news article with the echo-chamber information [17] using a tensor factorization approach. Using this method, our model is capable enough to classify using both the textual as well as user-community information. A deep neural network with five dense layers with a different number of filters across each layer is employed for the classification utilizing available tensor-based information. This method can be very helpful to discriminate the fake news with real ones with more efficient results with optimal hyperparameters.

\section{Methodology for fake news detection}

In this section, the complete methodology adopted in this research has been discussed in details. Our proposed method with tensor factorization approach is shown in Fig. 3.

\subsection{Mathematical representations}

In this research, scalar is represented by (e.g. $a$ ), matrix is represented by (e.g. $A$ ), and tensor is represented by boldfaced capital letter (e.g. $\boldsymbol{A}$ ).

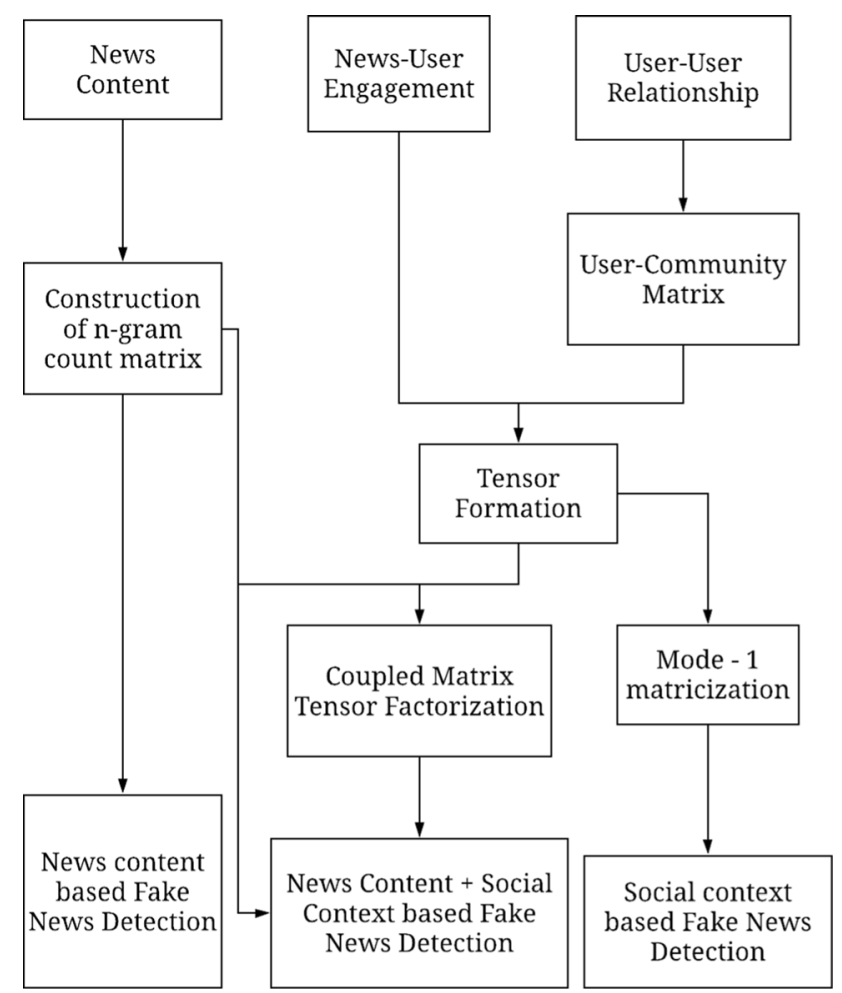

Fig. 3 Proposed method

\subsection{Construction of count matrix using textual content}

The matrix is represented by $N$ having the dimensions of $(n \times v)$, where $n$ is the total number of news articles in the dataset and $v$ is the number of words in the vocabulary. This matrix is used to count the sequences of words in a news article.

\subsection{Construction of news-user engagement matrix}

In this matrix, the response of the user to a particular news article has been shown. The matrix is denoted by $U$ having the dimensions of $(n \times u)$, where $n$ is the total number of news articles and $u$ is the number of users. This matrix is used to represent the counting of news articles shared by any particular user on social media.

\subsection{Development of user-community matrix}

A heuristic method for community detection in large scale networks is reported in the work of Blondel et al. [39], which uses an agglomerative multistep process during its execution. Wakita et al. [40] have also discussed community detection for small networks. In our research, the useruser relationship has been considered in the available 
information to build the user-community matrix. The method suggested by Clauset et al. [41] has been used for the fast identification of communities. This algorithm is a computationally resource-efficient and designed for large and complex networks. It associates each node of the network with a community. The computational complexity of the algorithms is analysed for the development of a deep neural network without compromising the performance of the model. In this algorithm, the main step is combining two communities that mainly contribute to the global modularity. Clauset et al. have proposed a matrix $M$ to store a modularity gain by the union of two communities $C_{a}$ and $C_{b}$ when the communities are connected. The elements $M_{a b}$ of Matrix $M$ are initialized by Eq. 1 . Here, $\mathrm{d}$ is a number which stores the sum of degrees of the modes in a network that belongs to a particular community (refer Eq. 2). User-community matrix is represented with $C$ having the dimensions of $(u \times c)$, where $u$ is the number of users in the social network and $c$ is the number of detected communities. According to Clauset, we have to update the matrix $M$ for each union until we get no more gain in the modularity. Clauset also defined the rules for updating (refer Eq. 3) the whole matrix $M$ with respect to connected communities which are being combined to other communities.

$M_{a b}= \begin{cases}\frac{1}{2 m}-\frac{d_{a} d_{b}}{(2 m)^{2}}, & \text { if } C_{a} \text { and } C_{b} \text { are connected } \\ 0, & \text { otherwise }\end{cases}$

Degree of vertex $a=d_{a}=\sum_{i} k_{i}, v_{i} \epsilon C_{a}$

$M_{a c}^{\prime}= \begin{cases}M_{a c}+M_{b c}, & \text { if } C_{c} \text { is connected to } C_{a} \text { and } C_{b} \\ M_{b c}-2 \frac{d_{a}}{(2 m)} \frac{d_{c}}{(2 m)}, & \text { if } C_{c} \text { is connected to } C_{b} \text { but not to } C_{a} \\ M_{a c}-2 \frac{d_{b}}{(2 m)} \frac{d_{c}}{(2 m)}, & \text { if } C_{c} \text { is connected to } C_{a} \text { but not to } C_{b}\end{cases}$

Here, $m=$ number of edges in the network, $k=$ degree vector, $v=a$ vertex in the network, $d=a$ vector which stores the sum of degree of nodes.

\subsection{Formation of tensor}

A tensor is formed as shown in Eq. (4). The representation of a 3-mode tensor is shown in Fig. 4 which consists of a combination of different feature matrices.

$T_{i j k}=U_{i j} * C_{j k}$

With the help of above tensor, the propagation of a news article in a community is represented.

\subsection{Re-ordering of tensor using matricization}

A tensor can be re-ordered into a matrix using matricization operation [25]. We can represent a mode- $i$ tensor $\boldsymbol{T}$ such that $\boldsymbol{T} \in R^{I_{1} \times I_{2} \cdots \times I_{i}}$. The mode-i matricization of the tensor $\boldsymbol{T}$ has been represented by Eq. (5).

$X_{i} \in R^{I_{i} \times\left(\prod_{n \neq i}^{3} I_{n}\right)}$

Here, the matrix $X_{1}$ represents mode-1 matricization having dimension $n \times(u * c)$. Here, $i$ is defined in the range of $[1,3]$.

\subsection{A coupled matrix-tensor factorization approach}

The combined representation of both news content as well as social context is fused by employing coupled matrixtensor factorization (CMTF) method [17, 42]. This approach solves the optimization, as stated in Eq. (6).

$\min \frac{1}{2}\left\|\boldsymbol{T}-\left[\left[T_{1}, T_{2}, T_{3}\right]\right]\right\|_{F}^{2}+\frac{1}{2}\left\|N-\left[\left[N_{1}, N_{2}\right]\right]\right\|_{F}^{2}$

In the Eq. (6), $\boldsymbol{T}$ is the 3-mode tensor (news, user and community). [ $\left.\left[T_{1}, T_{2}, T_{3}\right]\right]$ represents the Kruskal operation on matrices $T_{1}, T_{2}$ and $T_{3}$, such that $T_{1} \in R^{I_{1} \times R}, T_{2} \in R^{I_{2} \times R}$ and $T_{3} \in R^{I_{3} \times R}$. These matrices can be obtained by factorizing the tensor using the R-component PARAFAC procedure [43]. In the equation, $N$ denotes the news content matrix and $N_{1}$ and $N_{2}$ are the R-factor matrices obtained after nonnegative matrix factorization [44] of $N$, where $N_{1} \in R^{n \times R}$ and $N_{2} \in R^{v \times R}$. Equation (6) can be re-written as Eq. (7).

$\min \frac{1}{2} f_{1}+\frac{1}{2} f_{2}$

Optimization problem can be solved by computing gradients of the components $f_{1}$ and $f_{2}$ with respect to factors and shown with the help of Eqs. (8)-(10).

$\frac{\partial f_{1}}{\partial T_{i}}=\left(Z_{i}-X_{i}\right) T_{i}^{-i}$

$\frac{\partial f_{2}}{\partial N_{1}}=-N N_{2}+N_{1}^{-1} N_{2}^{T} N_{2}$

$\frac{\partial f_{2}}{\partial N_{2}}=-N^{T} N_{1}+N_{2} N_{1}^{T} N_{1}$

where

$$
\begin{aligned}
& Z=\left[\left[T_{1}, T_{2}, T_{3}\right]\right] \\
& Z_{1}=T_{1}\left(T_{3} \odot T_{2}\right)^{T}
\end{aligned}
$$


Fig. 4 Tensor decomposition approach
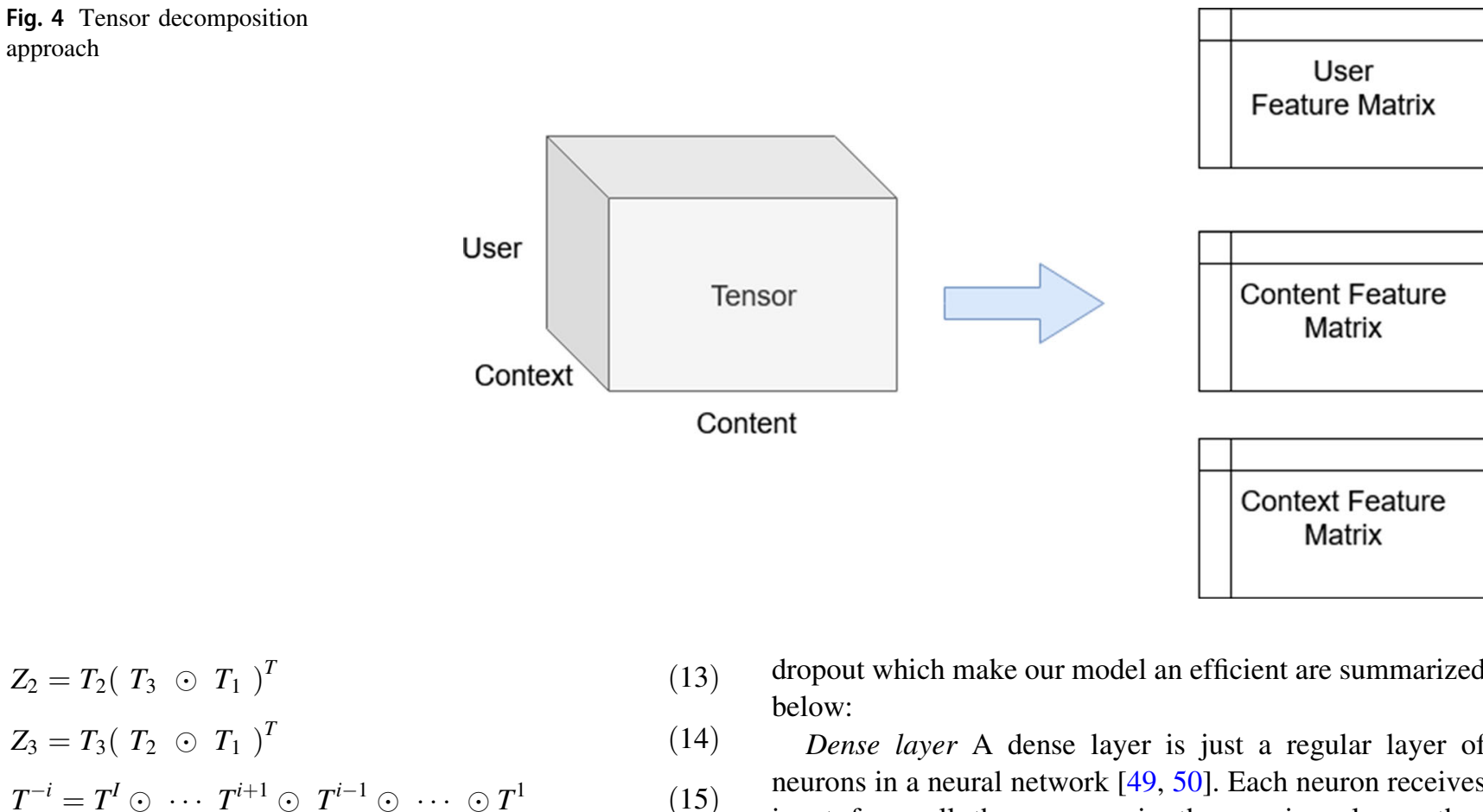

The symbol $\odot$ in Eqs. (12-15) represents Khatri-Rao product [45]. The final gradient matrix can be obtained by the concatenation of vectorized partial derivatives with respect to factor matrices as expressed in Eq. (16).

$$
\nabla_{f}=\left[\begin{array}{c}
\operatorname{vec}\left(\frac{\partial f_{1}}{\partial T_{1}}\right) \\
\operatorname{vec}\left(\frac{\partial f_{1}}{\partial T_{2}}\right) \\
\operatorname{vec}\left(\frac{\partial f_{1}}{\partial T_{3}}\right) \\
\operatorname{vec}\left(\frac{\partial f_{2}}{\partial N_{1}}\right) \\
\operatorname{vec}\left(\frac{\partial f_{2}}{\partial N_{2}}\right)
\end{array}\right]
$$

\section{Approach for fake news detection}

For the context of fake news detection [46-48], our proposed deep learning-based approach has been discussed in this section.

EchoFakeD: an efficient deep neural network In this research, we have designed a deep neural network with a diverse number of filters across each dense layer with dropout. In this network, we have considered five dense layers to make our model more effective and deep in nature. The selection of activation function, loss function, and

dropout which make our model an efficient are summarized below:

Dense layer A dense layer is just a regular layer of neurons in a neural network $[49,50]$. Each neuron receives input from all the neurons in the previous layer, thus densely connected. In our approach, we have taken five dense layers to make our model deep in nature with the selection of optimal hyperparameters.

Dropout Dropout is a regularization technique [49, 50], which is efficient and helpful to reduce the complexity of any classification model. It is also helpful to prevent overfitting [50]. In our research, we have applied the dropout at each layer of the network. This functionality has shown good results. We have conducted our experiments with the value of dropout to be 0.2 .

Activation function In our deep learning model, we have investigated using rectified linear unit (ReLU) [51] as the activation function. It is capable enough to remove negative values from an activation map by setting them to zero in a given network. The biggest advantage of ReLu is indeed non-saturation of its gradient, which greatly accelerates the convergence of stochastic gradient descent compared others activation functions [51]. It is also computationally efficient than sigmoid or tanh activation functions and solves the vanishing gradient problem. We can define the equation of ReLU as:

$\sigma=\max (0, z)$

Loss function $(L)$ Cross-entropy is widely used as a loss function for classification problems, because it minimizes the distance between two probability distributions-predicted and actual. The cross-entropy goes down as the prediction gets more and more accurate. It becomes zero if the prediction is perfect. Cross-entropy loss $(M=2)$ can be defined as: 


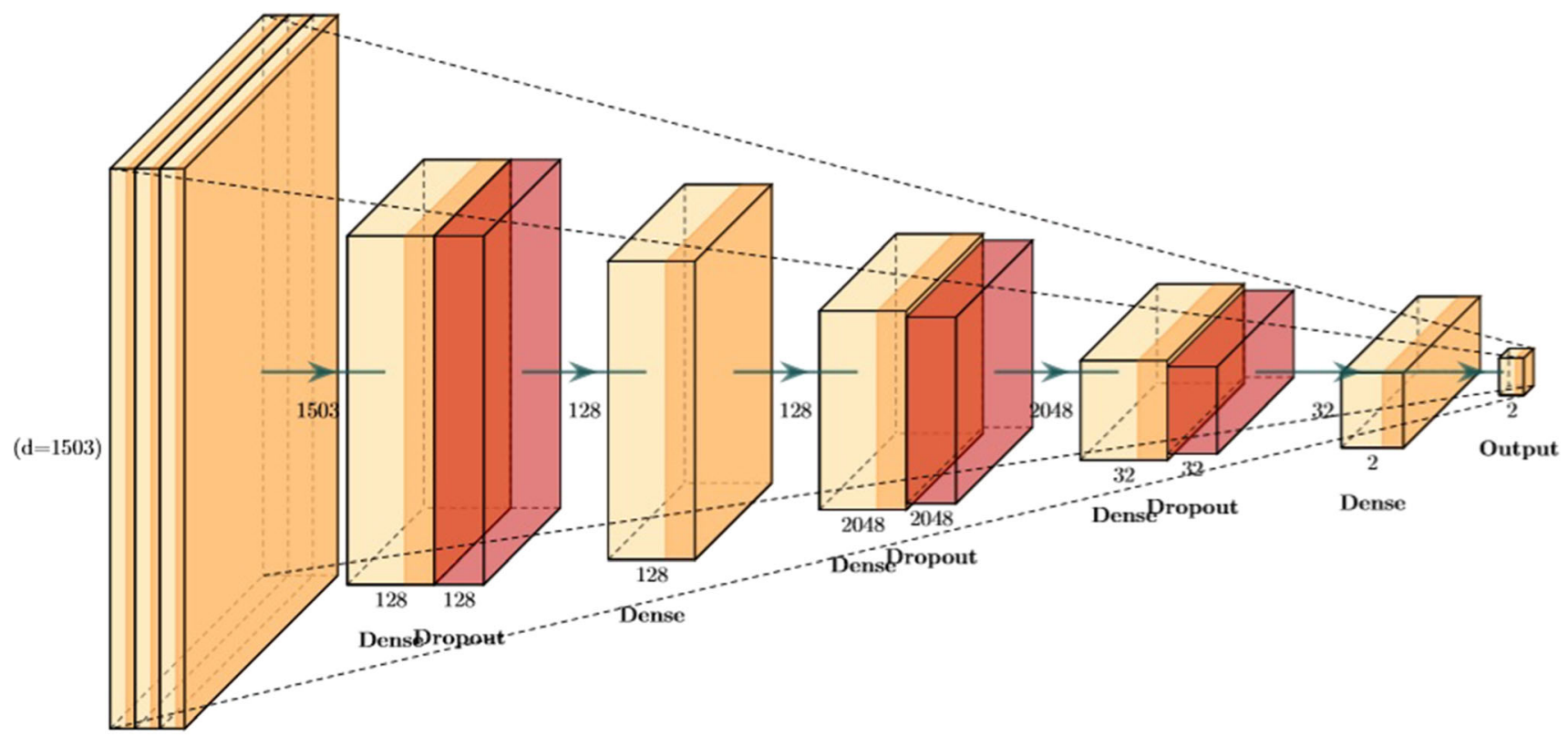

Input

Fig. 5 Architecture of our proposed network-EchoFakeD

$L=-(y \log (p)+(1-y) \log (1-p))$

If $M>2$ (i.e. multi-class classification), we calculate a separate loss for each class and sum the result.

$-\sum_{c=1}^{M} y_{o, c} \log \left(p_{o, c}\right)$

Here, $M$-number of classes, $c$ - a class, log-the natural logarithmic function, $y$-binary indicator ( 0 or 1$)$, $o$-an observation, $p$-predicted probability

In Fig. 5 and Table 1, the layered architecture of our proposed deep neural network (EchoFakeD) is shown. In this network, the input is based on word-embedding vectors with 1503 nodes. Our neural network consists of five dense layers. The first dense layer contains 128 hidden nodes with a dropout of 0.2 . The second dense layer contains 128 hidden nodes without dropout. The third dense layer

Table 1 Layered architecture of our proposed network-EchoFakeD

\begin{tabular}{lcc}
\hline Layer & Input (number of filters) & Output (number of filters) \\
\hline Dense layer & 1503 & 128 \\
Dropout layer & 128 & 128 \\
Dense layer & 128 & 128 \\
Dense layer & 128 & 2048 \\
Dropout layer & 2048 & 2048 \\
Dense layer & 2048 & 32 \\
Dropout layer & 32 & 32 \\
Dense layer & 32 & 2 \\
\hline
\end{tabular}

contains 2048 hidden nodes with a dropout of 0.2 . The fourth dense layer contains 32 hidden nodes with a dropout of 0.2 . The fifth dense layer contains 32 hidden nodes with a dropout of 0.2 . The final layer has 2 nodes with an activation function as SoftMax. The research work was carried using the NVIDIA DGX v100 machine, equipped with 40600 CUDA cores, 5120 tensor cores, 128 GB RAM, and 1000 TFLOPS speed.

\section{Experiments and results}

To benchmark our method, we have considered several classification approaches. These methods are based on the news article's textual content, user-context, and user-based relations.

\subsection{Dataset}

Experiments have been conducted to validate the performance of our proposed model using real-world fake news dataset: BuzzFeed and PolitiFact from the FakeNewsNet. ${ }^{3}$ The number of news articles and users in the fake news dataset is tabulated in Table 2 for PolitiFact and BuzzFeed. We have taken 145 news articles for training and 37 for testing the model (80:20 ratio). We have also validated the performance of our model using 37 fake news articles. The useful information in the dataset as follows:

\footnotetext{
${ }^{3}$ https://www.kaggle.com/mdepak/fakenewsnet.
} 
Table 2 FakeNewsNet dataset

\begin{tabular}{llcl}
\hline News source & News articles & Fake news articles & Number of users \\
\hline BuzzFeed & 182 & 91 & 15,257 \\
PolitiFact & 240 & 120 & 23,865
\end{tabular}

- News content: Having the attributes as news-id, URL, title, text, authors, and source of news.

- News-User engagement: It contains the information that how many times a news article has been shared by a user on social media.

- User-user engagement: It contains the relationships between the users.

\subsection{Feature extraction and hyperparameter setting}

\subsubsection{Feature extraction}

In this research, we have considered content, context, and user-community-based features for fake news classification. The Sklearn library is used to construct the features matrices. The dimensions of all the matrices (used as input features) are shown in Table 3. We have extracted 81 communities (featured as Echo chamber) using the Clauset-Newman-Moore algorithm [41].

\subsubsection{Hyperparameter setting}

Hyperparameters [52-54] can be defined as major elements or variables for a learning algorithm during the process of training and testing of any classification model. There exist two main approaches for selecting and optimizing the context-specific hyperparameters as a manual and automatic selection. The decision of selecting hyperparameters typically represents a trade-off between the manually versus automatic selection (in which the high computational cost is required). In our approach (for more details refer to Table 4), we have set the values of hyperparameters before training and optimizing the weights and bias.

\subsection{Performance parameters}

To validate the performance of our proposed model, different performance parameters have been considered: precision, recall, accuracy, and confusion matrix as evaluation matrices.

\subsubsection{Confusion matrix}

The information about the actual and predicted samples can be represented with the help of a confusion matrix. For binary classification, a confusion matrix is shown with the help of Table 5 .

\subsubsection{Precision and recall}

Recall is defined as:

Recall $=\frac{\mathrm{TP}}{\mathrm{TP}+\mathrm{FN}}$

where precision is :

Precision $=\frac{\mathrm{TP}}{\mathrm{TP}+\mathrm{FP}}$

\subsection{3 $F_{1}$-score}

$F_{1}$ score is the harmonic mean of Precision and Recall.

$F_{1}=\frac{2 *(\text { Precision } * \text { Recall })}{(\text { Precision }+ \text { Recall })}$

\subsubsection{Accuracy}

Accuracy is defined as:

$$
\text { Accuracy }=\frac{\mathrm{TP}+\mathrm{TN}}{\mathrm{TP}+\mathrm{TN}+\mathrm{FP}+\mathrm{FN}} \times 100
$$

Here $(\mathrm{TP})=$ correctly identified instances, $(\mathrm{FP})=$ incor rectly identified instances, $(\mathrm{TN})=$ correctly rejected instances, $(\mathrm{FN})=$ incorrectly rejected instances

\subsection{Experiments}

To classify the combination of both news content as well as social context-based information, tensor-based factorization method has been deployed. The order of classification tasks performed in our research as follows:

- EchoFakeD (our proposed deep neural network) with news content: For the experiment, the input feature matrix is the count matrix $N$.

- EchoFakeD with social context: For the experiment, social context-based are used. Matrix $\left(X_{1}\right)$ obtained after mode-1 matricization is used as input feature.

- EchoFakeD with news content and social context: For the experiment, we have used both news content as well as social context-based features for classification. Our proposed model has given state-of-the-art results with the combination of features. 
Table 3 Dimensionality of feature matrices

\begin{tabular}{ll}
\hline Matrix & Dimension \\
\hline News-user engagement matrix $(U)$ & $(182 \times 15,257)$ \\
Count matrix $(N)$ & $(182 \times 1500)$ \\
User-community matrix $(C)$ & $(15,257 \times 81)$ \\
Tensor $(T)$ & $(182 \times(15,257 \times 81)$ \\
Mode-1 tensor $\left(X_{1}\right)$ & $(182 \times(15,257 \times 81))$ \\
Input matrix $($ content + context $)$ & $(182 \times 1503)$ \\
\hline
\end{tabular}

Table 4 Hyperparameters for EchoFakeD

\begin{tabular}{ll}
\hline Hyperparameter & Value \\
\hline Number of dense layers & 5 \\
Number of hidden nodes & $128,128,32,2$ \\
Activation function & ReLU \\
Loss function & Binary cross-entropy \\
Optimizer & Adam \\
Dropout & 0.2 \\
Learning rate & 0.1 \\
Number of epochs & 20 \\
Batch-size & 64 \\
\hline
\end{tabular}

Table 5 Representation of confusion matrix

\begin{tabular}{lll}
\hline & Predicted positive & Predicted negative \\
\hline Actual positive & True positive (TP) & False negative (FN) \\
Actual negative & False positive (FP) & True negative (TN) \\
\hline
\end{tabular}

\subsection{Experimental results}

Experiments have been conducted using our proposed deep learning classifier (EchoFakeD) with different learning paradigms. From Fig. 5, we can observe the architectures of our proposed deep neural network. Classification results demonstrate that features selection and classification model plays an important role in the detection of fake news. In this research, a real-world fake news dataset (FakeNewsNet) has been used for classification.

Further, experiments have been conducted using our proposed deep neural network using both contents as well as social context of news articles. Tables 8 and 11 show that the combination of features gives more accurate results by employing a deep neural network. Respective confusion
Table 6 Confusion matrix for news content-based classification with EchoFakeD (BuzzFeed)

\begin{tabular}{lll}
\hline & Predicted positive & Predicted negative \\
\hline Actual positive & $17(\mathrm{TP})$ & $4(\mathrm{FN})$ \\
Actual negative & $3(\mathrm{FP})$ & $16(\mathrm{TN})$ \\
\hline
\end{tabular}

matrices for the deep learning approaches are shown with the help of Tables $6,7,8,9,10$ and 11 . The elements of confusion matrices give the number of correct and incorrect classifications. Our proposed model gave a better performance as compared to existing benchmarks employing tensor factorization methods using deep learning.

To validate the performance of our proposed model with the existing methods, several performance parameters like precision, recall, $F 1$-Score, false-positive rate, false-negative rate, and accuracy have been considered. Complete classification results (using Politifact and BuzzFeed dataset) are tabulated in Tables 12 and 13. In Table 13, the results using different combinations (news content, social context, and content+context) are presented with our proposed approach. Among the content and social contextbased methods, our proposed model has achieved an accuracy of $86.84 \%$ and $89.19 \%$, respectively. Combining social-context and news-content features, our proposed model achieved a marginal improvement over the baseline methods with an accuracy of $92.30 \%$. With these results, we recommend the effectiveness of social context-based features for fake news classification.

In this research, considering the performance of all classifiers, we found that with our proposed deep architecture, we achieved a validation accuracy of $92.30 \%$ using PolitiFact dataset. From Figs. 6 and 7, we can observe that with our proposed deep neural network, the validation accuracy is high and cross-entropy loss is minimum using both real-world fake news dataset. Our proposed model achieved accuracy with $91.80 \%$ using BuzzFeed dataset (refer Fig. 7). To validate the performance of our model, more performance parameters have been included (falsepositive rate (FPR) and false-negative rate (FNR)). The false-positive rate is $9.52 \%$ and the false-negative rate is $13.64 \%$ with our proposed model using BuzzFeed dataset (refer Table 14 for more details). False-negative rate is just $13.04 \%$ and the false-negative rate is $9.52 \%$ with our proposed model using PolitiFact dataset (refer Table 15 for more details). Results motivate the researchers to use our proposed method-EchoFakeD in future for the classification of fake news in their research. 
Table 7 Confusion matrix for social context-based classification with EchoFakeD (BuzzFeed)

\begin{tabular}{lll}
\hline & Predicted positive & Predicted negative \\
\hline Actual positive & $18(\mathrm{TP})$ & $3(\mathrm{FN})$ \\
Actual negative & $2(\mathrm{FP})$ & $16(\mathrm{TN})$ \\
\hline
\end{tabular}

Table 8 Confusion matrix for news content + social context-based classification with EchoFakeD (BuzzFeed)

\begin{tabular}{lll}
\hline & Predicted positive & Predicted negative \\
\hline Actual positive & $19(\mathrm{TP})$ & $3(\mathrm{FN})$ \\
Actual negative & $2(\mathrm{FP})$ & $19(\mathrm{TN})$ \\
\hline
\end{tabular}

Table 9 Confusion matrix for news content-based classification with EchoFakeD (PoitiFact)

\begin{tabular}{lll}
\hline & Predicted positive & Predicted negative \\
\hline Actual positive & $17(\mathrm{TP})$ & $3(\mathrm{FN})$ \\
Actual negative & $2(\mathrm{FP})$ & $16(\mathrm{TN})$ \\
\hline
\end{tabular}

Table 10 Confusion matrix for social context-based classification with EchoFakeD (PoitiFact)

\begin{tabular}{lll}
\hline & Predicted positive & Predicted negative \\
\hline Actual positive & $17(\mathrm{TP})$ & $2(\mathrm{FN})$ \\
Actual negative & $2(\mathrm{FP})$ & $16(\mathrm{TN})$ \\
\hline
\end{tabular}

Table 11 Confusion matrix using content and context-based features with EchoFakeD (PoitiFact)

\begin{tabular}{lll}
\hline & Predicted positive & Predicted negative \\
\hline Actual positive & $19(\mathrm{TP})$ & $2(\mathrm{FN})$ \\
Actual negative & $3(\mathrm{FP})$ & $20(\mathrm{TN})$ \\
\hline
\end{tabular}

\subsection{Comparison with existing classification methods}

From Tables 16 and 17, a comparison between existing classification benchmarks with our proposed model (EchoFakeD) has been shown. Table 16 shows the classification results with BuzzFeed dataset and Table 17 shows the classification results with PolitiFact dataset. Our proposed deep neural network has shown higher accuracy among all existing benchmark. False-positive rate and the false-negative rate are also less with our proposed model. Existing studies have primarily focused on the news content-based analysis. The problem of fake news has been investigated with not only the content-based attributes but also the relationship between news article and user on social media. Our approach is one step ahead of the existing one. In our approach, we have investigated the problem of fake news with an efficient deep neural network using the feature-vectors receiving from coupled matrixtensor factorization method as a 3-mode tensor. In this method, a tensor is created using the social context of news articles with several existing communities in the network. This method improved the performance of fake news classification compared to the existing methods. Results further motivated us to use our deep neural network as compared to existing traditional methods for efficient results.

\subsection{Discussion}

In Fig. 8, an example of fake news is shown. In this paper, we have performed extensive feature set-based studies for the classification of fake news. News content-based methods primarily focus on extracting different features from fake news articles, including both content-based $(B)$ as well as style-based. Style-based methods mainly focus on the writing style of manipulators and creators $(A)$ for the context of fake news. It is evident that for efficient fake news detection, content-based methodologies are alone not sufficient. We need to investigate the fake news articles with social context-based methods. Social context-based methods deal with the relationship among users, news article, and related publishers. These methodologies are
Table 12 Performance of our proposed model with BuzzFeed

\begin{tabular}{lllll}
\hline Approach & Precision & Recall & $F 1$-score & Accuracy \\
\hline EchoFakeD with news content & 0.8500 & 0.8095 & 0.8293 & 0.8250 \\
EchoFakeD with social context & 0.8571 & 0.9000 & 0.8780 & 0.8718 \\
EchoFakeD with content + context & 0.9047 & 0.8636 & 0.8837 & 0.9180 \\
\hline
\end{tabular}


Table 13 Performance of our proposed model with PolitiFact

\begin{tabular}{lllll}
\hline Approach & Precision & Recall & $F 1$-score & Accuracy \\
\hline EchoFakeD with news content & 0.8500 & 0.8947 & 0.8718 & 0.8684 \\
EchoFakeD with social context & 0.8947 & 0.8947 & 0.8947 & 0.8919 \\
EchoFakeD with content + context & 0.8636 & 0.9048 & 0.8837 & 0.9230 \\
\hline
\end{tabular}
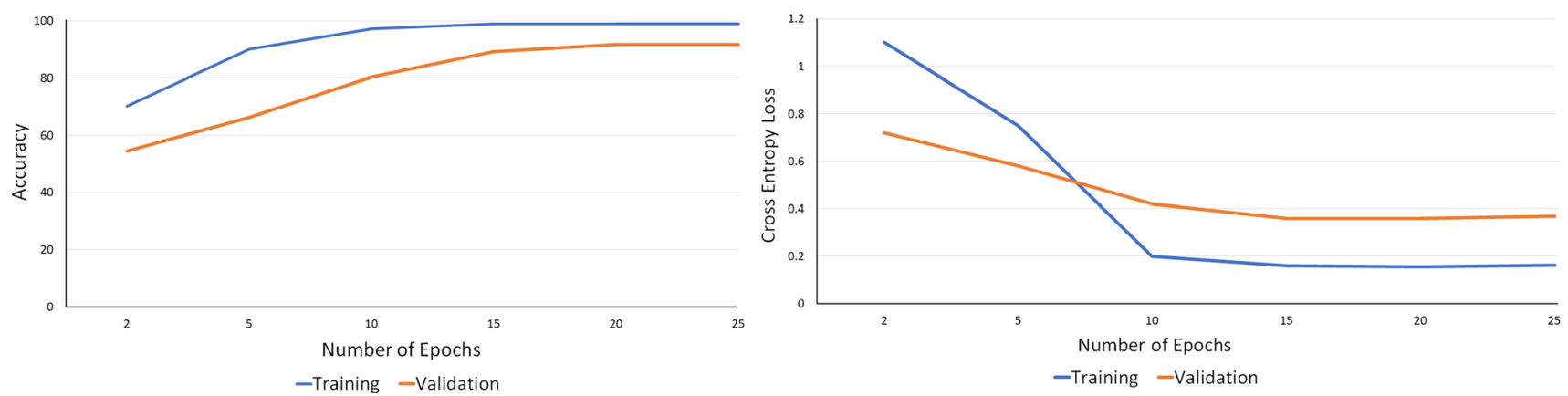

Fig. 6 Classification accuracy and cross-entropy loss with EchoFakeD using BuzzFeed
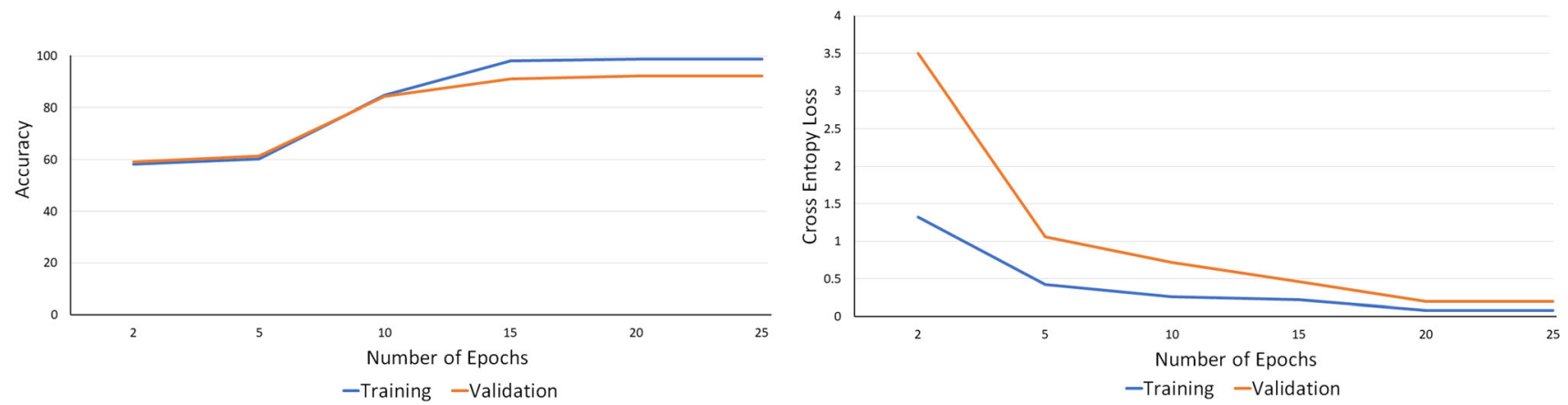

Fig. 7 Classification accuracy and cross-entropy loss with EchoFakeD using PolitiFact

Table 14 False-positive rate (FPR) and false-negative rate (FNR) using BuzzFeed

\begin{tabular}{lll}
\hline Approach & FPR & FNR \\
\hline EchoFakeD with news content & 0.1579 & 0.1905 \\
EchoFakeD with social context & 0.1111 & 0.1429 \\
EchoFakeD with content + context & 0.0952 & 0.1364 \\
\hline
\end{tabular}

Table 15 False-positive rate (FPR) and false-negative rate (FNR) using PolitiFact

\begin{tabular}{lll}
\hline Approach & FPR & FNR \\
\hline EchoFakeD with news content & 0.1111 & 0.1500 \\
EchoFakeD with social context & 0.1111 & 0.1053 \\
EchoFakeD with content + context & 0.1304 & 0.0952 \\
\hline
\end{tabular}

efficient to recognize fake news articles. Social context $(C)$ provides valuable information about users-based interaction with both the fake news as well as the real news. In the era of computing, at any social media platform, a user is always connected to a specific group of peoples having the same mindset or liking is called a user community $(D)$. These user communities can be an essential factor for fake news classification due to their common perception about sharing articles. Therefore, we have designed an effective deep neural network combining $(B+C+D)$ the content level features of news articles with user's social engagement (echo-chamber infused) to achieve significant results. Subsequently, the tensor factorization-based approach has been used with content as well as context-based information.

In Fig. 8, an example of fake news is shown. In this paper, we have performed extensive feature set-based studies for the classification of fake news. News contentbased methods primarily focus on extracting different features from fake news articles, including both contentbased $(B)$ as well as style-based. Style-based methods mainly focus on the writing style of manipulators and creators $(A)$ for the context of fake news. It is evident that for efficient fake news detection, content-based 
Table 16 Comparison with existing benchmarks with BuzzFeed

Table 17 Comparison with existing benchmarks using PolitiFact

Fig. 8 An example of fake news. Source: Facebook

\begin{tabular}{llcc}
\hline Authors & Precision $(\%)$ & Recall $(\%)$ & F1-score $(\%)$ \\
\hline Castillo et al. [55] & 73.50 & 78.30 & 75.60 \\
Castillo et al. [55] + RST & 79.50 & 78.40 & 78.90 \\
Gupta et al. (CITDetect) [17] & 65.70 & 100.00 & 79.20 \\
Gupta et al. (CIMTDetect) [17] & 72.90 & 92.30 & 81.30 \\
Papanastasiou et al. [56] (CLASS-CP) & 85.20 & 83.00 & 83.50 \\
Zhou et al. [35] & 84.90 & 85.20 & 84.20 \\
[38] (DNN-with echo chamber) & 83.33 & 86.96 & 85.11 \\
Proposed model-EchoFakeD & 90.47 & 86.36 & 88.37 \\
\hline
\end{tabular}

\begin{tabular}{llll}
\hline Authors & Precision $(\%)$ & Recall $(\%)$ & F1-score $(\%)$ \\
\hline Castillo et al. [55] & 77.70 & 79.10 & 78.30 \\
Castillo et al. [55] + RST & 82.30 & 79.20 & 79.30 \\
Gupta et al. (CITDetect) [17] & 67.90 & 97.50 & 79.10 \\
Gupta et al. (CIMTDetect ) [17] & 80.30 & 84.20 & 81.80 \\
Papanastasiou et al. [56] (CLASS-CP) & 87.20 & 82.10 & 84.30 \\
[38] (DNN-with echo chamber) & 82.10 & 84.60 & 84.04 \\
Proposed model-EchoFakeD & 86.36 & 90.48 & 88.37 \\
\hline
\end{tabular}

methodologies are alone not sufficient. We need to investigate the fake news articles with social context-based methods. Social context-based methods deal with the relationship among users, news articles, and related publishers. These methodologies are efficient in recognizing fake news articles. Social context $(C)$ provides valuable information about users-based interaction with fake news and real news. In the era of computing, at any social media platform, a user is always connected to a specific group of peoples having the same mindset or liking is called a usercommunity $(D)$. These user communities can be an essential factor for fake news classification due to their common perception about sharing articles. Therefore, we have designed an effective deep neural network combining $(B+C+D)$ the content level features of news articles with user's social engagement (echo-chamber infused) to 
achieve significant results. Subsequently, the tensor factorization-based approach has been used with content as well as context-based information. In this paper, a user's engagement with the news articles is captured and fused with user-community interaction to form a 3-mode tensor (content, social-context, and user-community information). This tensor is capable of handling multi-relational data and provides a higher dimensional generalization of matrices. Tensor factorization decomposes the higher-order tensor into low-rank tensors. The resulting low-rank tensors capture the complex relations between the objects representing the help of models of the tensor. Therefore, in this research, a coupled matrix-tensor factorization method is used with a latent representation of news articles' news content and context. In the coupled matrix-tensor factorization method (also known as CP-decomposition), we have used the standard factorization method to decompose the matrix. To validate the classification performance, the proposed deep learning model (EchoFakeD) is employed with both contents and context-based information. Our model outperformed existing and appropriate baselines for fake news detection and achieved an accuracy of $92.30 \%$.

\section{Conclusion and future scope}

A methodology utilizing both news content and socialcontext of news articles with the tensor-factorization method has presented. For an effective classification, a tensor factorization approach has presented with a deep neural network. The performance of our proposed approach is evaluated on the real-world fake news dataset: BuzzFeed and PolitiFact. A detailed analysis has presented utilizing different features: news content, social context, and the combination of news content and social context. With the classification results, it is clear that the combination approach using tensor factorization gives better performance, as shown in the form of evaluation parameters. Our proposed approach has improved the classification result in terms of $F 1$-score and validation accuracy compared to existing methods. Our future plan would be to perform a more accurate classification of fake news using the temporal information with content and social context-based features. For the task of fake news detection, a feature set can never be considered complete and sound. Therefore, in addition to temporal-based information $[7,8]$ would be helpful in fake news propagation. Another future work is also to address the issues in fake news detection using content, context, temporal, and echo chambers-based information. It would be interesting to explore new ways of representing the available information with tensors at a methodological level. As further work, we would like to explore the extent to which our model (EchoFakeD) improves when more features are added.

\section{Compliance with ethical standards}

Conflict of interest All the authors of this manuscript certify that they have NO affiliations with or involvement in any organization or entity with any financial interest (such as honoraria; educational grants; participation in speakers' bureaus; membership, employment, consultancies, stock ownership, or other equity interest; and expert testimony or patent licensing arrangements), or non-financial interest (such as personal or professional relationships, affiliations, knowledge or beliefs) in the subject matter or materials discussed in this manuscript.

\section{References}

1. Ahmed H, Traore I, Saad S (2017) Detection of online fake news using $\mathrm{N}$-gram analysis and machine learning techniques. In: International conference on intelligent, secure, and dependable systems in distributed and cloud environments. Springer, Cham, pp $127-138$

2. Weir W (2009) History's greatest lies: the startling truths behind world events our history books got wrong. Fair Winds Press, Beverly

3. De Vreese H, Peter J, Holli A, Semetko C (2001) Framing politics at the launch of the Euro: a cross-national comparative study of frames in the news. Polit Commun 18(2):107-122

4. Shu K, Mahudeswaran D, Liu H (2019) FakeNewsTracker: a tool for fake news collection, detection, and visualization. Comput Math Org Theory 25(1):60-71

5. McBeth MK, Clemons RS (2011) Is fake news the real news? In: Amarasingam A (ed) The Stewart/Colbert effect: essays on the real impacts of fake news. McFarland, California, pp 79-98

6. Miller TP, Leon A (2017) Introduction to special issue on literacy, democracy, and fake news: making it right in the era of fast and slow literacies. Lit Compos Stud 5(2):10-23

7. Kumar S, Shah N (2018) False information on web and social media: a survey. arXiv: arXiv-1804

8. Shin J, Jian L, Driscoll K, Bar F (2018) The diffusion of misinformation on social media: temporal pattern, message, and source. Comput Hum Behav 83:278-287

9. Zhou X, Zafarani R (2018) Fake news: a survey of research, detection methods, and opportunities. arXiv preprint arXiv:1812. 00315

10. Ghosh S, Shah C (2018) Towards automatic fake news classification. Proc Assoc Inf Sci Technol 55(1):805-807

11. Roy A, Basak K, Ekbal A, Bhattacharyya P (2018) A deep ensemble framework for fake news detection and classification. arXiv: arXiv-1811

12. Ruchansky N, Sungyong S, Liu Y (2017) Csi: a hybrid deep model for fake news detection. In: Proceedings of the 2017 ACM on conference on information and knowledge management. ACM, pp 797-806

13. Sharma K, Qian F, Jiang H, Ruchansky N, Zhang M, Liu Y (2019) Combating fake news: a survey on identification and mitigation techniques. ACM Trans Intell Syst Technol 10(3): $1-42$

14. Shu K, Mahudeswaran D, Wang S, Lee D, Liu H (2020) FakeNewsNet: a data repository with news content, social context, and 
spatiotemporal information for studying fake news on social media. Big Data 8(3):171-188

15. Vosoughi S, Mohsenvand MN, Roy D (2017) Rumor gauge: predicting the veracity of rumors on Twitter. ACM Trans Knowl Discov Data 11(4):1-36

16. Fazil M, Abulaish M (2018) A hybrid approach for detecting automated spammers in twitter. IEEE Trans Inf Forensics Secur 13(11):2707-2719

17. Gupta S, Thirukovalluru R, Sinha M, Mannarswamy S (2018) CIMTDetect: a community infused matrix-tensor coupled factorization based method for fake news detection. In: 2018 IEEE/ ACM international conference on advances in social networks analysis and mining (ASONAM). IEEE, pp 278-281

18. Ott M, Choi Y, Cardie C, Hancock JT (2011) Finding deceptive opinion spam by any stretch of the imagination. In: Proceedings of the 49th annual meeting of the association for computational linguistics: human language technologies, vol 1. Association for Computational Linguistics, pp 309-319

19. Feng S, Banerjee R, Choi Y (2012) Syntactic stylometry for deception detection. In: Proceedings of the 50th annual meeting of the association for computational linguistics: short papers, vol 2. Association for Computational Linguistics, pp 171-175

20. Chen Y, Conroy NJ, Rubin VL (2015) Misleading online content: recognizing clickbait as false news. In: Proceedings of the 2015 ACM on workshop on multimodal deception detection, pp 15-19

21. Pérez-Rosas V, Kleinberg B, Lefevre A, Mihalcea R (2018) Automatic detection of fake news. In: Proceedings of the 27th international conference on computational linguistics, pp 3391-3401

22. Gupta M, Zhao P, Han J (2012) Evaluating event credibility on twitter. In: Proceedings of the 2012 SIAM international conference on data mining. Society for Industrial and Applied Mathematics, pp 153-164

23. Shu K, Cui L, Wang S, Lee D, Liu H (2019) Defend: explainable fake news detection. In: Proceedings of the 25th ACM SIGKDD international conference on knowledge discovery and data mining, pp 395-405

24. Zhou X, Wu J, Zafarani R (2020) SAFE: similarity-aware multimodal fake news detection. arXiv: arXiv-2003

25. Rabanser S, Shchur O, Günnemann S (2017) Introduction to tensor decompositions and their applications in machine learning. arXiv: arXiv-1711

26. Hosseinimotlagh S, Papalexakis EE (2018) Unsupervised content-based identification of fake news articles with tensor decomposition ensembles. In: Proceedings of the workshop on misinformation and misbehavior mining on the web (MIS2)

27. Paisitkriangkrai S, Sherrah J, Janney $P$, Van-Den Hengel A (2015) Effective semantic pixel labelling with convolutional networks and conditional random fields. In: 2015 IEEE conference on computer vision and pattern recognition workshops (CVPRW). IEEE, pp 36-43

28. Zagoruyko S, Komodakis N (2015) Learning to compare image patches via convolutional neural networks. In: Proceedings of the IEEE conference on computer vision and pattern recognition, pp 4353-4361

29. Shu K, Wang S, Liu H (2019) Beyond news contents: the role of social context for fake news detection. In: Proceedings of the twelfth ACM international conference on web search and data mining. ACM, pp 312-320

30. Tacchini E, Ballarin G, Della Vedova ML, Moret S, de Alfaro L (2017) Some like it hoax: automated fake news detection in social networks. arXiv preprint arXiv:1704.07506

31. Zhang J, Dong B, Yu PS (2020) Fakedetector: effective fake news detection with deep diffusive neural network. In: 2020 IEEE 36th international conference on data engineering (ICDE). IEEE, pp 1826-1829
32. Ma J, Gao W, Mitra P, Kwon S, Jansen BJ, Wong K-F, Cha M (2016)Detecting rumors from microblogs with recurrent neural networks 3818

33. Yang Y, Zheng L, Zhang J, Cui Q, Li Z, Yu PS (2018) TI-CNN: convolutional neural networks for fake news detection. arXiv: arXiv-1806

34. Shu K, Wang S, Liu H (2019) Beyond news contents: the role of social context for fake news detection. In: Proceedings of the twelfth ACM international conference on web search and data mining, pp 312-320

35. Zhou X, Zafarani R (2019) Network-based fake news detection: a pattern-driven approach. ACM SIGKDD Explor Newsle 21(2):48-60

36. Wang Y, Ma F, Jin Z, Yuan Y, Xun G, Jha K, Su L, Gao J (2018) Eann: Event adversarial neural networks for multi-modal fake news detection. In: Proceedings of the 24th ACM SIGKDD international conference on knowledge discovery and data mining, pp 849-857

37. Kaliyar RK, Goswami A, Narang P, Sinha S (2020) FNDNet-a deep convolutional neural network for fake news detection. Cognit Syst Res 61:32-44

38. Kaliyar RK, Goswami A, Narang P (2020) DeepFakE: improving fake news detection using tensor decomposition-based deep neural network. J Supercomput

39. Blondel VD, Guillaume J-L, Lambiotte R, Lefebvre E (2008) Fast unfolding of communities in large networks. J Stat Mech Theory Exp 2008(10):P10008

40. Wakita K, Tsurumi T (2007) Finding community structure in mega-scale social networks. In: Proceedings of the 16th international conference on World Wide Web, pp 1275-1276

41. Clauset A, Newman MEJ, Moore C (2004) Finding community structure in very large networks. Phys Rev E 70(6):066111

42. Acar E, Kolda TG, Dunlavy DM (2011) All-at-once optimization for coupled matrix and tensor factorizations. arXiv: arXiv-1105

43. Harshman RA (1968) Foundations of the PARAFAC procedure: models and conditions for an "explanatory" multimodal factor analysis, vol 30, issue 2. Indian Statistical Institute, pp 1-84. https://www.jstor.org/stable/i25049520

44. Lee D, Seung HS (2001) Algorithms for non-negative matrix factorization. In: Advances in neural information processing systems, pp 556-562

45. Khatri CG, Radhakrishna RC (1968) Solutions to some functional equations and their applications to characterization of probability distributions. Sankhyā Indian J Stat Ser A 167-180

46. Chen T, Guestrin C (2016) Xgboost: a scalable tree boosting system. In: Proceedings of the 22nd ACM SIGKDD international conference on knowledge discovery and data mining, pp 785-794

47. Torlay L, Perrone-Bertolotti M, Thomas E, Baciu M (2017) Machine learning-XGBoost analysis of language networks to classify patients with epilepsy. Brain Inform 4(3):159-169

48. Natekin A, Knoll A (2013) Gradient boosting machines, a tutorial. Front Neurorobotics 7:21

49. Vasudevan V, Zoph B, Shlens J, Le QV (2019) Neural architecture search for convolutional neural networks. U.S. Patent 10,521,729, issued December 31

50. Zhong B, Xing X, Love P, Wang X, Luo H (2019) Convolutional neural network: deep learning-based classification of building quality problems. Adv Eng Inform 40:46-57

51. Li Y, Yuan Y (2017) Convergence analysis of two-layer neural networks with relu activation. In: Advances in neural information processing systems, pp 597-607

52. Djidjev HN (2006) A scalable multilevel algorithm for graph clustering and community structure detection. In: Aiello W, Broder A, Janssen J, Milios E (eds) International workshop on algorithms and models for the web-graph. Springer, Berlin, pp $117-128$ 
53. MacKay DJC (1999) Comparison of approximate methods for handling hyperparameters. Neural Comput 11(5):1035-1068

54. He K, Zhang X, Ren S, Sun J (2015) Delving deep into rectifiers: surpassing human-level performance on imagenet classification. In: Proceedings of the IEEE international conference on computer vision, pp 1026-1034

55. Castillo Carlos, Mendoza Marcelo, Poblete Barbara (2011) Information credibility on twitter. In Proceedings of the 20th international conference on World wide web, pp. 675-684
56. Papanastasiou F, Katsimpras G, Paliouras G (2019) Tensor factorization with label information for fake news detection. arXiv: arXiv-1908

Publisher's Note Springer Nature remains neutral with regard to jurisdictional claims in published maps and institutional affiliations. 